$\mathrm{J}$ o u r n a l of

Mathematics

and Applications

JMA No 38, pp 77-84 (2015)

\title{
Some results on 2-absorbing ideals in commutative semirings
}

\author{
Pratibha Kumar, Manish Kant Dubey \\ and Poonam Sarohe
}

\begin{abstract}
In this paper, we study the concepts of 2-absorbing and weakly 2-absorbing ideals in a commutative semiring with non-zero identity which is a generalization of prime ideals of a commutative semiring and prove number of results related to the same. We also use these concepts to prove some results of $Q$-ideals in terms of subtractive extension of ideals in a commutative semiring.
\end{abstract}

AMS Subject Classification: 16Y30, 16 Y60

Keywords and Phrases: Semiring, subtractive ideal, 2-absorbing ideal, weakly 2-absorbing ideal, subtractive extension of an ideal, Q-ideal.

\section{Introduction}

The semiring is an important algebraic structure which plays a prominent role in various branches of mathematics as well as in diverse areas of applied science. The concepts of semiring was first introduced by H. S. Vandiver [14] in 1934. After that several authors have apllied this concept in various disciplines in many ways. The structure of prime ideals in semiring theory has gained importance and many mathematicians have exploited its usefulness in algebraic systems over the decades. Anderson and Smith[3] introduced the notion of weakly prime ideals in commutative ring. The concept of 2-absorbing and weakly 2 -absorbing ideals of commutative ring with non-zero unity have been introduced by Badawi [5] and Badawi and Darani[6] respectively which are generalizations of prime and weakly prime ideals in a commutative ring. Darani[8] has explored these concepts in commutative semiring and characterized several results in terms of 2-absorbing and weakly 2-absorbing ideals in commutative semiring. Chaudhary and Bonde[7] have introduced the notion of subtractive extension of an ideal to study the ideal theory in quotient semiring.

A commutative semiring is a commutative semigroup $(S, \cdot)$ and a commutative monoid $\left(S,+, 0_{S}\right)$ in which $0_{S}$ is the additive identity and $0_{S} \cdot x=x \cdot 0_{S}=0_{S}$ for

COPYRIGHT (c) by Publishing Department Rzeszów University of Technology P.O. Box 85, 35-959 Rzeszów, Poland 
all $x \in S$, both are connected by ring like distributivity. A nonempty subset $I$ of a semiring $S$ is called an ideal of $S$ if $a, b \in I, r \in S, a+b \in I$ and $r a, a r \in I$. An ideal $I$ of a semiring $S$ is called subtractive if $a, a+b \in I, b \in S$ then $b \in I$. An ideal $(I: r)$ is defined as $(I: r)=\{x \in S: r x \in I\}$. It is easy to see that if $I$ is a subtractive ideal of $S$, then $(I: r)$ is a subtractive ideal of $S$. Radical of an ideal $I$ is defined as $\operatorname{Rad}(I)=\sqrt{I}=\left\{a \in S: a^{n} \in I\right.$ for some positive integer $\left.n\right\}$. An element $s$ in a semiring $S$ is said to be nilpotent if there exists a positive integer $n$ (depending on $s)$ such that $s^{n}=0 . \operatorname{Nil}(S)$ denotes the set of all nilpotent element of $S$. A proper ideal $I$ of a semiring $S$ is said to be prime (respectively, weakly prime) if $a b \in I$ (respectively, $0 \neq a b \in I$ ) implies $a \in I$ or $b \in I$ for some $a, b \in S$. An ideal $I$ of a semiring $S$ is said to be irreducible if for ideals $H$ and $K$ of $S, I=H \cap K$ implies that $I=H$ or $I=K$. A semiring $S$ is said to be regular if for each $a \in S$ there exists $x \in S$ such that $a=a x a$. In [11], it is proved that a semiring $S$ is regular if and only if $H K=H \cap K$ for all left ideals $K$ and right ideals $H$ of $S$.

Throughout this paper, $S$ will always denote a commutative semiring with identity $1 \neq 0$.

\section{$2 \quad$ 2-absorbing and weakly 2-absorbing ideals}

In this section, we prove number of results correspond to 2-absorbing and weakly 2-absorbing ideals in commutative semirings. Recall [8] the following definitions.

Definition 2.1. A proper ideal I of a commutative semiring $S$ is said to be a 2absorbing ideal of $S$ if whenever $a, b, c \in S$ and $a b c \in I$, then $a b \in I$ or $a c \in I$ or $b c \in I$.

Definition 2.2. A proper ideal I of $S$ is said to be a weakly 2-absorbing ideal of $S$ if whenever $a, b, c \in S$ and $0 \neq a b c \in I$, then $a b \in I$ or $a c \in I$ or $b c \in I$.

It is easy to see that every 2-absorbing ideal of a semiring $S$ is a weakly 2 -absorbing ideal of $S$ but converse need not be true. For further understanding properties of 2absorbing and weakly 2 -absorbing ideals in commutative semirings, refer [8].

Theorem 2.3. Let $I$ be a 2-absorbing ideal of $S$. Then $(I: r)$ is a 2-absorbing ideal of $S$ for all $r \in S \backslash I$.

Proof. Let $r \in S \backslash I$ and let $a, b, c \in S$ be such that $a b c \in(I: r)$. Then $r a b c \in I$. So $r a \in I$ or $r b c \in I$ or $a b c \in I$, since $I$ is a 2-absorbing ideal of $S$. If either $r a \in I$ or $r b c \in I$, we are done. If $a b c \in I$, then $a b \in I$ or $a c \in I$ or $b c \in I$, which implies $r a b \in I$ or $r a c \in I$ or $r b c \in I$. Hence $(I: r)$ is a 2-absorbing ideal of $S$.

Theorem 2.4. Let $I$ be a 2-absorbing subtractive ideal of $S$ with $\sqrt{I}=J$ and $J^{2} \subseteq I$. If $I \neq J$ and for all $r \in J \backslash I$, then $(I: r)$ is a prime ideal of $S$ containing $I$ with $J \subseteq(I: r)$.

Proof. Let $u v \in(I: r)$ for some $u, v \in S$. Then $r u v \in I$. Since $I$ is a 2-absorbing ideal of $S$, therefore $r u \in I$ or $r v \in I$ or $u v \in I$. If $r u \in I$ and $r v \in I$, then $u \in(I: r)$ 
or $v \in(I: r)$, therefore nothing to prove. If $u v \in I$ and also, $r^{2} \in J^{2} \subseteq I$. This gives $r v \in(I: r)$ for particular $v \in S$. We have $(r+u) v \in(I: r)$, that is, $r(r+u) v \in I$ and since $I$ is a 2-absorbing ideal of $S$, therefore $r v \in I$ or $(r+u) v \in I$ or $r(r+u) \in I$. If $r v \in I$ then $v \in(I: r)$, which is required. If $(r+u) v \in I$ and $u v \in I$, then $r v \in I$ (as $I$ is a subtractive). This gives $v \in(I: r)$, so $(I: r)$ is prime. Finally, if $r(r+u) \in I$ and since $r^{2} \in J^{2} \subseteq I$. This gives $r u \in I$ implies $u \in(I: r)$. Hence $(I: r)$ is a prime ideal of $S$.

Corollary 2.5. Let $I$ be a 2-absorbing subtractive ideal of $S$ with $\sqrt{I}=J$ and $J^{2} \subseteq I$. If $I \neq J$ and for all $r \in J \backslash I$, then $(I: r)$ is a 2-absorbing ideal of $S$ with $J \subseteq(I: r)$.

Theorem 2.6. If $I$ is a subtractive ideal of $S$ such that $I \neq \sqrt{I}$ and $\sqrt{I}$ is a prime ideal of $S$ with $(\sqrt{I})^{2} \subset I$. Then $I$ is a 2-absorbing ideal of $S$ if and only if $(I: r)=$ $\{x \in S: r x \in I\}$ is a prime ideal of $S$ for each $r \in \sqrt{I} \backslash I$.

Proof. $(\Rightarrow)$ One way is straight forward by above theorem.

$(\Leftarrow)$ Conversely, let $a b c \in I$ for some $a, b, c \in S$. Then, we may assume that $a \in \sqrt{I}$ (as $I \subseteq \sqrt{I}$ and $\sqrt{I}$ is a prime ideal of $S$ ). If $a \in I$, then $a b \in I$, which gives $I$ is a 2-absorbing ideal of $S$. Assume that $a \in \sqrt{I} \backslash I$. Also, $b c \in(I: a)$ and by assumption $(I: a)$ is a prime ideal of $S$, therefore we have either $b \in(I: a)$ or $c \in(I: a)$. This implies that either $a b \in I$ or $a c \in I$. Thus, $I$ is a 2-absorbing ideal of $S$.

The following result is used to prove the next theorem.

Result 2.7. [12] Let $I$ and $J$ be two subtractive ideals in $S$. Then $I \cup J$ is a subtractive ideal of $S$ if and only if $I \cup J=I$ or $I \cup J=J$.

Theorem 2.8. Let $I$ be a 2-absorbing subtractive ideal of $S$ with $\sqrt{I}=J$. If $I \neq J$, $J$ is a prime ideal of $S$ and for all $r \in S \backslash J$, then $\Omega=\{(I: r): r \in S\}$ is a totally ordered set.

Proof. Let $r, s \in S \backslash J$. Since $J$ is a prime ideal of $S$ therefore $r s \in S \backslash J$. Clearly, $r s \notin I$ and $(I: r) \subseteq(I: r s)$ and $(I: s) \subseteq(I: r s)$ which implies $(I: r) \cup(I: s) \subseteq(I: r s)$. Again, let $t \in(I: r s)$. Then, $r s t \in I$ which implies that either $r t \in I$ or $s t \in I$, as $r s \notin I$. Thus, $(I: r s) \subseteq(I: r) \cup(I: s)$. Hence by Result 2.7, we have either $(I: r s)=(I: r)$ or $(I: r s)=(I: s)$. This implies that either $(I: r) \subseteq(I: s)$ or $(I: s) \subseteq(I: r)$. Therefore $\Omega=\{(I: r): r \in S \backslash J\}$ is a totally ordered set.

Again, we show that $(I: s) \subseteq(I: r)$ for $r, s \in J \backslash I$. Let $r, s \in J \backslash I$. Then for any $p \in(I: r) \backslash(I: s)$ we may assume that $p \in(I: r) \backslash J$, since $J \subseteq(I: s)$. Similarly, for any $q \in(I: s) \backslash(I: r)$ we may assume that $q \in(I: s) \backslash J$. Since $p \notin J$ and $q \notin J$ therefore $p q \notin J$. Also, $p(r+s) q \in I$ and $p q \notin I$, therefore we have $p(r+s) \in I$ or $(r+s) q \in I$, which gives either $p s \in I$ or $r q \in I$. This implies $p \in(I: s)$ or $q \in(I: r)$. Therefore, in each case we get a contradiction. Hence either $(I: r) \subseteq(I: s)$ or $(I: s) \subseteq(I: r)$ for $r, s \in J \backslash I$. Thus, $\Omega=\{(I: r): r \in S\}$ is a totally ordered set. 
Theorem 2.9. Let $I$ be an irreducible subtractive ideal of $S$ and let $J$ be an ideal of $S$ such that $\sqrt{I}=J$ and $J^{2} \subseteq I$. Then $I$ is 2-absorbing if and only if $(I: r)=\left(I: r^{2}\right)$ for all $r \in S \backslash J$.

Proof. Let $I$ be a 2-absorbing ideal of $S$. For $r \in S \backslash J, r^{2} \notin I$ because if $r^{2} \in I$, then $r \in \sqrt{I}=J$, which is a contradiction and also $(I: r) \subseteq\left(I: r^{2}\right)$ is obvious. So, for any $s \in\left(I: r^{2}\right)$ we have $r^{2} s \in I$. Since $I$ is a 2- absorbing ideal of $S$, we have either $r s \in I$ or $r^{2} \in I$. Since $r^{2} \notin I$, therefore $r s \in I$, that is, $s \in(I: r)$ and thus $(I: r)=\left(I: r^{2}\right)$.

Conversely, let $r s t \in I$ for some $r, s, t \in S$ and $r s \notin I$. We show that either $r t \in I$ or $s t \in I$. From $r s \notin I$, we have $r \notin J$ or $s \notin J$. Because, if $r \in J$ and $s \in J$, then $r s \in J^{2} \subseteq I$, a contradiction. Now, by assumption, we have either $(I: r)=\left(I: r^{2}\right)$ or $(I: s)=\left(I: s^{2}\right)$. If $(I: r)=\left(I: r^{2}\right)$ and also assume that $r t \notin I$ and $s t \notin I$, then we prove the result by way of contradiction. Let $p \in(I+(r t)) \cap(I+(s t))$. Then there are $p_{1}, p_{2} \in I$ and $r_{1}, r_{2} \in S$ such that $p=p_{1}+r_{1} r t=p_{2}+r_{2} s t$. Thus, $p r=p_{1} r+r_{1} r^{2} t=p_{2} r+r_{2} r s t \in I$. Since $r s t \in I$, therefore $r_{1} r^{2} t \in I$ (as $I$ is a subtractive ideal of $S)$. This implies $r_{1} r t \in I$ because $(I: r)=\left(I: r^{2}\right)$. Hence $p=p_{1}+r_{1} r t \in I$. This shows that $(I+r t) \cap(I+s t) \subseteq I$ and thus $(I+r t) \cap(I+s t)=I$, a contradiction because $I$ is an irreducible. Thus, we have $r t \in I$ or $s t \in I$ and consequently, $I$ is a 2 -absorbing ideal of $S$.

Theorem 2.10. Let $S$ be a regular semiring. Then every irreducible ideal I of $S$ is 2-absorbing ideal of $S$.

Proof. Let $S$ be a regular semiring and $I$ be an irreducible ideal of $S$. If $r s t \in I$ and $r s \notin I$, then we have to show that $r t \in I$ or $s t \in I$. On contrary, we assume that $r t \notin I$ and $s t \notin I$. Then, $H=(I+(r t))$ and $K=(I+(s t))$ be two ideals of $S$ properly contain $I$. Since $I$ is an irreducible, therefore $I \neq H \cap K$. Thus, there exists $p \in S$ such that $p \in(I+(r t)) \cap(I+(s t)) \backslash I$. Also, by regularity of $S$, we have $H \cap K=H K$, therefore $p \in\left(I+(r t)\left(I+(s t) \backslash I\right.\right.$. Then, there are $p_{1}, p_{2} \in I$ and $r_{1}, r_{2} \in S$ such that $p=\left(p_{1}+r_{1} r t\right)\left(p_{2}+r_{2} s t\right)=p_{1} p_{2}+p_{1} r_{2} s t+r_{1} r t p_{2}+r s r_{1} r_{2} t^{2}$. This implies that $p \in I$, which is a contradiction. Hence $I$ is a 2-absorbing ideal of $S$.

Proposition 2.11. Let $a \in S$ and $I$ be an ideal of $S$. Then the following holds:

(i) If $S a$ is a subtractive ideal of $S$ and $(0: a) \subseteq S a$, then the ideal Sa is 2-absorbing if and only if it is weakly 2-absorbing.

(ii) If $I$ is a subtractive ideal of $S$ and $(0: a) \subseteq I a$, then the ideal Ia is 2-absorbing if and only if it is weakly 2-absorbing.

Proof. (i). Let $S a$ be weakly 2-absorbing ideal of $S$ and $r s t \in S a$ for some $r, s, t \in S$. If $r s t \neq 0$, then $r s \in S a$ or $s t \in S a$ or $r t \in S a$. Then we have done. Assume that $r s t=0$. Clearly, $r(s+a) t=r s t+r a t \in S a$. If $r(s+a) t \neq 0$, then $r(s+a) \in S a$ or $r t \in S a$ or $(s+a) t \in S a$ (as $S a$ is a weakly 2-absorbing ideal of $S$ ). Hence $r s \in S a$ or $s t \in S a$ or $r t \in S a$, since $S a$ is a subtractive ideal of $S$. So, assume that $r(s+a) t=0$. 
Since $r s t=0$, therefore we have $r a t=0$ and so $r t \in(0: a) \subseteq S a$. Thus, $r t \in S a$. Hence $S a$ is a 2 -absorbing ideal of $S$.

(ii). The proof is similar to (i)

Theorem 2.12. ([8], Theorem 2.6) Let $S$ be a commutative semiring. If I is a weakly 2-absorbing subtractive ideal of $S$, then either $I^{3}=0$ or $I$ is 2-absorbing.

The above theorem is used to prove the next theorem which is a generalization of ([6], Theorem 2.7).

Theorem 2.13. Let $I$ be a weakly 2-absorbing subtractive ideal of $S$ but not a 2absorbing ideal of $S$. Then

(i) if $r \in \operatorname{Nil}(S)$, then either $r^{2} \in I$ or $r^{2} I=r I^{2}=\{0\}$.

(ii) $\operatorname{Nil}(S)^{2} I^{2}=\{0\}$.

Proof. (i). Let $r \in \operatorname{Nil}(S)$. We claim that if $r^{2} I \neq\{0\}$. Then $r^{2} \in I$. Suppose that $r^{2} I \neq\{0\}$. Let $n$ be the least positive integer such that $r^{n}=0$, then for $n \geq 3$ and for some $s \in I$, we have $0 \neq r^{2} s=r^{2}\left(s+r^{n-2}\right) \in I$. Since $I$ is a weakly 2-absorbing ideal of $S$, we have either $r^{2} \in I$ or $\left(r s+r^{n-1}\right) \in I$. If $r^{2} \in I$, we have nothing to prove. Let $r^{2} \notin I$. Then $\left(r s+r^{n-1}\right) \in I$, which gives $r^{n-1} \in I$ and $r^{n-1} \neq 0$, and thus $r^{2} \in I$. Hence for each $r \in \operatorname{Nil}(S)$ we have either $r^{2} \in I$ or $r^{2} I=\{0\}$. If $s^{2} \notin I$ for some $s \in \operatorname{Nil}(S)$, then by previous argument, we have $s^{2} I=\{0\}$. We claim that $s I^{2}=\{0\}$. Suppose that $s i_{1} i_{2} \neq 0$ for some $i_{1}, i_{2} \in I$. Let $m \geq 3$ be the least positive integer such that $s^{m}=0$. Since $s^{2} \notin I, m \geq 3$ and $s^{2} I=\{0\}$, therefore $s\left(s+i_{1}\right)\left(s^{m-2}+i_{2}\right)=s i_{1} i_{2} \neq 0$. Since $0 \neq s\left(s+i_{1}\right)\left(s^{m-2}+i_{2}\right) \in I$ and $I$ is a weakly 2-absorbing ideal of $S$, we have either $s^{2} \in I$ or $0 \neq s^{m-1} \in I$ (as $I$ is a subtractive ideal of $S$ ). Therefore, we have $s^{2} \in I$, a contradiction. Hence $s I^{2}=\{0\}$.

(ii). Let $a, b \in \operatorname{Nil}(S)$. If either $a^{2} \notin I$ or $b^{2} \notin I$, then by part (i), we have $a b I^{2}=\{0\}$ and hence the result. For $a^{2} \in I$ and $b^{2} \in I$, then $a b(a+b) \in I$. If $0 \neq a b(a+b) \in I$ and since $I$ is a subtractive weakly 2-absorbing ideal of $S$, we have $a b \in I$. So by Theorem 2.12, we have $a b I^{2}=\{0\}$. Again, if $0=a b(a+b) \in I$ and $0 \neq a b i \in I$ for some $i \in I$, then $0 \neq a b(a+b+i) \in I$ implies either $a(a+b+i) \in I$ or $b(a+b+i) \in I$ or $a b \in I$. In each case, we have $a b \in I$, which is a contradiction, as $I$ is a weakly 2 -absorbing and not a 2 -absorbing ideal of $S$. Thus, we have $a b I=\{0\}$ and hence $a b I^{2}=\{0\}$.

Definition 2.14 ( [4], Definition 1(i) ). A proper ideal I of $S$ is called strong ideal, if for each $a \in I$ there exists $b \in I$ such that $a+b=0$.

Proposition 2.15. Let $S$ and $S^{\prime}$ be semirings, $f: S \mapsto S^{\prime}$ be an epimorphism such that $f(0)=0$ and $I$ be a subtractive strong ideal of $S$. Then the following holds:

(i). If $I$ is a weakly 2-absorbing ideal of $S$ such that ker $f \subseteq I$, then $f(I)$ is a weakly 2-absorbing ideal of $S^{\prime}$.

(ii). If $I$ is a 2-absorbing ideal of $S$ such that ker $f \subseteq I$, then $f(I)$ is a 2-absorbing ideal of $S^{\prime}$. 
Proof. (i). Let $a, b, c \in S^{\prime}$ be such that $0 \neq a b c \in f(I)$. Then there exists $n \in I$ such that $0 \neq a b c=f(n)$. Since $f$ is an epimorphism, therefore there exist $p, q, r \in S$ such that $f(p)=a, f(q)=b, f(r)=c$. Also, since $I$ is a strong ideal of $S$ and $n \in I$, then there exists $m \in I$ such that $n+m=0$. This implies $f(n+m)=0$, that is, $f(p q r+m)=0$, implies, $p q r+m \in k e r f \subseteq I$. So, $0 \neq p q r \in I$ (as $I$ is subtractive) because if $p q r=0$, then $f(n)=0$, a contradiction. Since $I$ is a weakly 2-absorbing ideal of $S$, therefore either $p q \in I$ or $q r \in I$ or $r p \in I$. Thus, $a b \in f(I)$ or $b c \in f(I)$ or $c a \in f(I)$. Therefore, $f(I)$ is a weakly 2-absorbing ideal of $S^{\prime}$.

(ii). It follows from (i).

Consider $S=S_{1} \times S_{2}$ where each $S_{i}$ is a commutative semiring with unity, $i=1,2$ with $\left(a_{1}, a_{2}\right)\left(b_{1}, b_{2}\right)=\left(a_{1} b_{1}, a_{2} b_{2}\right)$ for all $a_{1}, b_{1} \in S_{1}$ and $a_{2}, b_{2} \in S_{2}$.

Proposition 2.16. If $I$ is a proper ideal of a semiring $S_{1}$. Then the following statements are equivalent:

(i). I is a 2-absorbing ideal of $S_{1}$.

(ii). $I \times S_{2}$ is a 2-absorbing ideal of $S=S_{1} \times S_{2}$.

(iii). $I \times S_{2}$ is a weakly 2-absorbing ideal of $S=S_{1} \times S_{2}$.

Proof. $(i) \Rightarrow(i i)$. Let $\left(a_{1}, a_{2}\right),\left(b_{1}, b_{2}\right),\left(c_{1}, c_{2}\right) \in S$ be such that $\left(a_{1}, a_{2}\right)\left(b_{1}, b_{2}\right)\left(c_{1}, c_{2}\right) \in$ $I \times S_{2}$. Then $\left(a_{1} b_{1} c_{1}, a_{2} b_{2} c_{2}\right) \in I \times S_{2}$. Therefore, $a_{1} b_{1} c_{1} \in I$. This gives either $a_{1} b_{1} \in I$ or $b_{1} c_{1} \in I$ or $c_{1} a_{1} \in I$, since $I$ is a 2-absorbing ideal of $S_{1}$. If $a_{1} b_{1} \in I$, then $\left(a_{1}, a_{2}\right)\left(b_{1}, b_{2}\right) \in I \times S_{2}$. Similarly, we can prove the other cases. Hence, $I \times S_{2}$ is a 2-absorbing ideal of $S$.

$($ ii $) \Rightarrow($ iii $)$. It is obvious.

$($ iii $) \Rightarrow(i)$. Let $a b c \in I$ for some $a, b, c \in S_{1}$. Then for each $0 \neq r \in S_{2}$, we have $(0,0) \neq(a, 1)(b, 1)(c, r) \in I \times S_{2}$. This gives, either $(a, 1)(b, 1) \in I \times S_{2}$ or $(b, 1)(c, r) \in I \times S_{2}$ or $(c, r)(a, 1) \in I \times S_{2}$, since $I \times S_{2}$ is a weakly 2-absorbing ideal of $S$. That is, either $a b \in I$ or $b c \in I$ or $c a \in I$. This shows that $I$ is a 2-absorbing ideal of $S_{1}$.

Definition 2.17 ([1], Definition(4)). An ideal I of a semiring $S$ is called a $Q$-ideal (partitioning ideal) if there exists a subset $Q$ of $S$ such that

(i) $S=\cup\{q+I: q \in Q\}$

(ii) If $q_{1}, q_{2} \in Q$, then $\left(q_{1}+I\right) \cap\left(q_{2}+I\right) \neq \emptyset \Leftrightarrow q_{1}=q_{2}$.

Let $I$ be a $Q$ - ideal of a semiring $S$. Then $S / I_{(Q)}=\{q+I: q \in Q\}$ forms a semiring under the following addition ' $\oplus$ ' and multiplication ' $\odot$ ', $\left(q_{1}+I\right) \oplus\left(q_{2}+I\right)=q_{3}+I$ where $q_{3} \in Q$ is unique such that $q_{1}+q_{2}+I \subseteq q_{3}+I$, and $\left(q_{1}+I\right) \odot\left(q_{2}+I\right)=q_{4}+I$ where $q_{4} \in Q$ is unique such that $q_{1} q_{2}+I \subseteq q_{4}+I$. This semiring $S / I_{(Q)}$ is called the quotient semiring of $S$ by $I$ and denoted by $\left(S / I_{(Q)}, \oplus, \odot\right)$ or just $S / I_{(Q)}$. By definition of $Q$-ideal, there exists a unique $q_{0} \in Q$ such that $0+I \subseteq q_{0}+I$. Then $q_{0}+I$ is a zero element of $S / I_{(Q)}$. Clearly, if $S$ is commutative then so is $S / I_{(Q)}$. 
Definition 2.18 ([7], Definition(2.4)). Let I be an ideal of a semiring $S$. An ideal $A$ of $S$ with $I \subseteq A$ is said to be subtractive extension of $I$ if $x \in I, x+y \in A, y \in S$, then $y \in A$.

Further, we give some characterizations of 2-absorbing and weakly 2-absorbing ideals in terms of subtractive extension of an ideal of a semiring $S$, which are derived from generalizations of [7].

Theorem 2.19. Let $S$ be a semiring, $I$ be a $Q$-ideal of $S$ and $P$ a subtractive extension of $I$. Then $P$ is 2-absorbing ideal of $S$ if and only if $P / I_{(Q \cap P)}$ is a 2-absorbing ideal of $S / I_{(Q)}$.

Proof. Let $P$ be a 2-absorbing ideal of $S$. Suppose that $q_{1}+I, q_{2}+I, q_{3}+I \in S / I_{(Q)}$ are such that $\left(q_{1}+I\right) \odot\left(q_{2}+I\right) \odot\left(q_{3}+I\right)=q_{4}+I \in P / I_{(Q \cap P)}$ where $q_{4} \in Q \cap P$ is a unique element such that $q_{1} q_{2} q_{3}+I \subseteq q_{4}+I \in P / I_{(Q \cap P)}$. So $q_{1} q_{2} q_{3}=q_{4}+i$ for some $i \in I$. Since $P$ is a 2-absorbing ideal of $S$ and $q_{1} q_{2} q_{3} \in P$, therefore $q_{1} q_{2} \in P$ or $q_{2} q_{3} \in P$ or $q_{3} q_{1} \in P$. Consider the case $q_{1} q_{2} \in P$. If $\left(q_{1}+I\right) \odot\left(q_{2}+I\right)=i_{1}+I$ where $i_{1} \in Q$ is a unique element such that $q_{1} q_{2}+I \subseteq i_{1}+I$. So $i_{1}+f=q_{1} q_{2}+e$ for some $e, f \in I$. Since $P$ is a subtractive extension of $I$, we have $i_{1} \in P$, therefore $i_{1} \in Q \cap P$. Hence $P / I_{(Q \cap P)}$ is a 2-absorbing ideal of $S / I_{(Q)}$.

Conversely, if $P / I_{(Q \cap P)}$ is a 2-absorbing ideal of $S / I_{(Q)}$. Let $a b c \in P$ for some $a, b, c \in S$. Since $I$ is a $Q$-ideal of $S$, therefore there exist $q_{1}, q_{2}, q_{3}, q_{4} \in Q$ such that $a \in q_{1}+I, b \in q_{2}+I, c \in q_{3}+I$ and $a b c \in\left(q_{1}+I\right) \odot\left(q_{2}+I\right) \odot\left(q_{3}+I\right)=q_{4}+I$. So, $a b c=q_{4}+i_{2} \in P$ for some $i_{2} \in I$. Since $P$ is a subtractive extension of $I$, we have $q_{4} \in P$. So $\left(q_{1}+I\right) \odot\left(q_{2}+I\right) \odot\left(q_{3}+I\right)=q_{4}+I \in P / I_{(Q \cap P)}$, which gives $\left(q_{1}+I\right) \odot\left(q_{2}+I\right) \in P / I_{(Q \cap P)}$ or $\left(q_{2}+I\right) \odot\left(q_{3}+I\right) \in P / I_{(Q \cap P)}$ or $\left(q_{3}+I\right) \odot\left(q_{1}+I\right) \in P / I_{(Q \cap P)}$, since $P / I_{(Q \cap P)}$ is a 2-absorbing ideal of $S / I_{(Q)}$. If $\left(q_{1}+I\right) \odot\left(q_{2}+I\right) \in P / I_{(Q \cap P)}$, then there exists $q_{5} \in Q \cap P$ such that $a b \in$ $\left(q_{1}+I\right) \odot\left(q_{2}+I\right)=q_{5}+I$. This gives $a b=q_{5}+i_{3}$ for some $i_{3} \in I$. This implies $a b \in P$. Thus, $P$ is a 2 -absorbing ideal of $S$.

Corollary 2.20. Let $S$ be a semiring, $I$ be a $Q$-ideal of $S$ and $P$ be subtractive ideal of $S$ such that $I \subseteq P$. Then $P$ is a 2-absorbing ideal of $S$ if and only if $P / I_{(Q \cap P)}$ is a 2-absorbing ideal of $S / I_{(Q)}$.

Note that, if $\left(q_{1}+I\right) \odot\left(q_{2}+I\right) \odot\left(q_{3}+I\right) \neq 0$ in $S / I_{(Q)}$, then $q_{1} q_{2} q_{3} \neq 0$ in $S$. Now one can easily prove the next theorem, adopting the proof of the last theorem.

Theorem 2.21. Let $S$ be a semiring, $I$ a $Q$-ideal of $S$ and $P$ a subtractive extension of $I$. Then

(i) $f P$ is a weakly 2-absorbing ideal of $S$, then $P / I_{(Q \cap P)}$ is a weakly 2-absorbing ideal of $S / I_{(Q)}$.

(ii) if $I$ and $P / I_{(Q \cap P)}$ is a weakly 2-absorbing ideal of $S$ and $S / I_{(Q)}$ respectively, then $P$ is a weakly 2-absorbing ideal of $S$. 


\section{References}

[1] Allen, P. J.: A fundamental theorem of homomorphism for semirings, Proc. Amer. Math. Soc., 21(1969), $412-416$.

[2] Anderson, D. D., Bataineh, M.: Generalizations of prime ideals, Comm. Algebra, 36(2),(2008), $686-696$.

[3] Anderson, D. D. and Smith, Eric: Weakly prime ideals, Houston J. Math., 29 (2003), $831-840$.

[4] Atani, R. E. and Atani, S. E.: Spectra of semimodules; Bul. Acad. De Stiinte, A Republicii Moldova. Math., 3(67), (2011), $15-28$.

[5] Badawi, A.: On 2-absorbing ideals of commutative rings, Bull Austral. Math. Soc., $75(2007), 417-429$.

[6] Badawi, A. and Darani, A. Y.: On weakly 2-absorbing ideals of commutative rings, Houston J. Math., 39(2), (2013), 441 - 452.

[7] Chaudhari, J. N. and Bonde, D. R.: Ideal theory in quotient semirings, Thai J. Of Math., Vol.12, (2014), $95-101$.

[8] Darani, A. Y.: On 2-absorbing and weakly 2-absorbing ideals of commutative Semirings, Kyungpook Math. J., 52(1), (2012), $91-97$.

[9] Dubey, M. K.: Prime and weakly prime ideals in semirings, Quasigroups and Related Systems, 20 (2012), $151-156$.

[10] Dubey, M. K. and Sarohe, P.: On 2-absorbing semimodules, Quasigroups and Related Systems, 21 (2013), 175-184.

[11] Golan, J. S. : Semirings and their applications, Kluwer Academic Publishers, Dordrecht, (1999).

[12] Gupta,V. and Chaudhari, J. N.: Characterization of weakly prime subtractive ideals in semirings, Bull. Inst. Math. Acad. Sin. (N.S.), 3(2) (2008), 347 - 352.

[13] Payrovi, Sh. and Babaei, S.: On the 2-absorbing ideals in commutative rings, Bull. Malaysian Math. Sci., 23, (2013), 1511-1526.

[14] Vandiver H. S.: Note on a Simple type of Algebra in Which the Cancellation Law of Addition Does Not Hold, Bull. Amer. Math. Soc., 40(3), (1934) 916 - 920.

DOI: $10.7862 / \mathrm{rf} .2015 .7$

\section{Pratibha Kumar}

email: pratibhakumar313@gmail.com

Department of Mathematics, Kirori Mal College, University of Delhi, Delhi 110007, India.

\section{Manish Kant Dubey}

email: kantmanish@yahoo.com

SAG, DRDO, Metcalf House, Delhi 110054, India.

\section{Poonam Sarohe- corresponding author}

email: poonamsarohe@gmail.com

Department of Mathematics, Lakshmibai College, University of Delhi, Delhi 110052, India.

Received 16.05.2014 